$4 \times 3 \mathrm{~cm}$ diameter mass located in the inferior vermis and 4 th ventricle advancing to foramen magnum.

Conclusion Swallowing difficulties in children is generally due to mild problems such as gastroesophageal reflux, esophagitis or food allergies. If swallowing difficulty is together with weight loss and is persistant for months intracranial pathologies, as in our case, should be searched.

\section{INSIDE THE MIND OF AN ANGEL}

doi:10.1136/archdischild-2012-302724.0543

R Kyeremateng. Department of Paediatrics, Royal Cornwall Hospital, Truro, UK

Background Angelman syndrome is a neurogenetic disorder characterised by severe learning difficulties and speech impairment, motor difficulties including jerky movements and ataxia, and seizures.

Epilepsy associated with Angelman syndrome consists of a variety of seizure types. Typically EEG shows a distinctive pattern which can aid diagnosis, but MRI scan shows no abnormality.

Although there is a known association between epilepsy and hippocampal sclerosis in the general population, the development of hippocampal sclerosis following a prolonged convulsion has not been described in a child with Angelman syndrome.

Methodology Literature search was carried out to review and compare similar reported cases. This elucidated that the association between hippocampal sclerosis and Angelman syndrome has rarely been cited.

Results The case presented here is a 3 year old girl with Angelman syndrome due to de novo micro deletion of chromosome 15, who suffered a prolonged convulsion and subsequently developed a persistent hemiplegia. Serial MRI scans demonstrate initially normal brain architecture and appearances, then the evolution from mild hippocampal swelling two days after the acute insult, to frank hippocampal sclerosis, as well as changes to the left cerebral hemisphere, several months later.

Conclusion This case evidences the development of hippocampal sclerosis following acute prolonged convulsion in a child with Angelman syndrome, and implicates this pathogenesis in the natural history of Angelman syndrome.

\section{BENIGN EXTRA AXIAL COLLECTION OF INFANCY - A CASE REPORT}

doi:10.1136/archdischild-2012-302724.0544

A Kage. Paediatrics, Kettering General Hospital, Kettering, UK

Benign extra axial collection of infancy is a benign clinical entity characterized by rapid head enlargement in an infant with normal neurodevelopment.

We report on an infant who was referred at 3 months with rapidly increasing head circumference. The development was normal and there was a family history of macrocephaly. MRI brain showed normal ventricles with no hydrocephalus. There was significant prominence of subarachnoid space, particularly in fronto- parietal regions. Interestingly, there was moderate degree of cerebral atrophy.

Paediatricians should consider this diagnosis in any infant with rapid head enlargement and normal neurodevelopment. It is a benign condition that requires no surgical intervention because it often resolves spontaneously. The age of onset varies, but it is often seen in the first year of life, more often in boys, when an infant is noticed to have rapid head enlargement. It should not be confused with hydrocephalus or any other intracranial pathologies that are often associated with abnormal neurodevelopmental milestones. The persistence of the subarachnoid fluid collection beyond 2 years of age or a change in neurodevelopment calls for further evaluation to exclude intracranial pathology.

\section{VICI SYNDROME ASSOCIATED WITH SENSORINEURAL HEARING LOSS AND LARYNGOMALASIA}

doi:10.1136/archdischild-2012-302724.0545

${ }^{1} \mathrm{M}$ Özkale, ${ }^{2 i}$ Erol, ${ }^{3} \mathrm{~A}$ Gümüş, 'Y Özkale, 'B Kilıçaslan, ${ }^{2} \mathrm{~F}$ Alehan. ${ }^{1}$ Department of Pediatrics; 'Department of Pediatrics, Division of Child Neurology; ${ }^{3}$ Department of Pediatrics, Division of Cardiology, Baskent University Hospital, Adana, Turkey

Background and Aims Vici syndrome is characterized by albinism, hypopigmentation, agenesis of the corpus callosum, catarats, immundeficeny, recurrent severe infections, hipertrophic cardiomyopathy and psychomotor retardation. To the best of our knowledge, this is the first reported case of a Turkish patient with Vici syndrome. Case Report A 3 months-old girl admitted to our hospital for bronchopneumonia, stridor and failure to thrive. Physical examination revealed marked hypopigmentation of the skin with silvery hair, and dysmorphic features including highed-arched palate, micrognathia, generalize hypotonia, truncal ataxia with absense of deep-tendon reflexes (Figure 1). Ophthalmological examination revealed bilateral anterior subcapsular cataracs, and ocular albinism. Metabolic screening was normal. Magnetic resonance imaging of the brain showed agenesis of corpus callosum together with delayed myelinisation of cerebral white matter and hypoplasia of the cerebellar hemisphere and brainstem (Figure 2). Echocardiography was demonstrated hypertrophic cardiomyopathy. Odiological examination showed deafness on the left ears. Direct laryngoscopy was performed due to stridor and revealed laryngomalasia. She had immunological abnormalities including, decreased CD3+ (\%38.1), CD3+/CD4+ (\%31.1), CD3+/CD8+ (\%7.2). Ceftriaxon and $\beta$-blocker were given for bronchopneumonia and cardiomyopathy. She had been lost due to broncopnomonia in an other hospital at the age of 6 months.

Conclusion Vici syndrome is considered in the different diagnosis of infants presenting with congenital agenesis of the corpus callosum. As until now 14 patients with Vici syndrome were reported we want to draw attention to this rare syndrome.

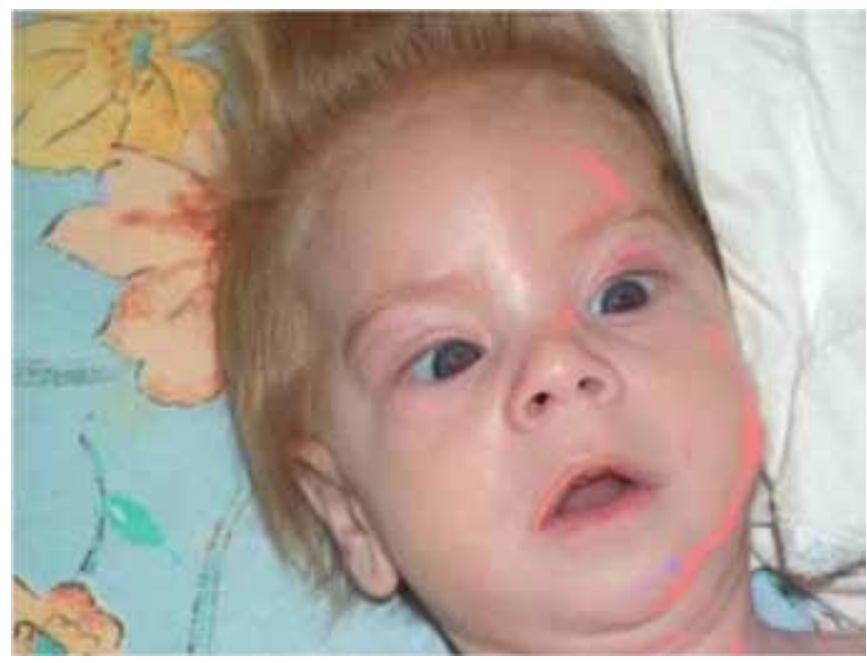

Abstract 545 Figure 1 